\title{
Leptomeningeal Carcinomatosis of Metastatic Primary Prostate Cancer
}

\author{
Daniel Stenberg $^{a} \quad$ Megha Shalavadi $^{a} \quad$ Humera Syeda ${ }^{a} \quad K^{2}$ rishna Reddy ${ }^{a}$ \\ Phillip Saunders ${ }^{b}$ \\ aUHS Southern California Medical Education Consortium, Temecula, CA, USA; \\ bThe Oncology Institute of Hope and Innovation, Temecula, CA, USA
}

\section{Highlights}

- $\quad$ Leptomeningeal carcinomatosis continues to be a diagnostic challenge due to limited and varying sensitivities of diagnostic modalities used. However, the advances in MRI gadolinium imaging should place its findings equivalent or superior to lumbar puncture in terms of consistency, sensitivity, and risk to the patient.

- $\quad$ Prognosis remains poor despite advances in therapies due to limited evidence from studies and variable/limited response to treatment that is limited by toxicity.

- Understanding the molecular mechanisms of metastasis to the brain may help detect and evaluate better, focused therapies targeting tumor-specific molecular markers across different tumor types.

- $\quad$ Early and prompt diagnosis will prolong life. Patients with hematologic or solid tumors who have clinical symptoms of leptomeningeal carcinomatosis warrant screening with routine cranial imaging.

\section{Keywords}

Leptomeningeal carcinomatosis · Lumbar puncture · Prostate cancer metastasis

\begin{abstract}
Leptomeningeal carcinomatosis (LC) is an uncommon complication of cancer in which the disease metastasizes to the meninges; it is estimated that this occurs in $5 \%$ of cancer patients and is most often terminal. LC has a median survival time of approximately 15.7 weeks [Leal et al., Curr Cancer Ther Rev. 2011;7(4):319-27]. Furthermore, metastasis from the prostate is exceptionally rare with only a few cases described in medical literature. Until recently, leptomeningeal disruption was very rare and cerebral involvement was irrelevant [Bubendorf et al., Hum Pathol. 2000;31(5):578-83; Schaller et al., Br J Cancer. 2000;77(12):2386-9]. With improved imaging, diagnostic modalities, and treatment with therapies that do not cross the bloodbrain barrier, the incidence of LC has been on the rise [Batool and Kasi, StatPearls, https://
\end{abstract}

\section{Karger ${ }^{\prime \prime}$}


www.ncbi.nlm.nih.gov/books/NBK499862/]. Diagnosis previously relied on biopsy, supported by lumbar puncture findings. We submit a case demonstrating progressive and consistent evidence of LC found on imaging, justifying its acceptance as a diagnostic modality.

(C) 2021 The Author(s).

Published by S. Karger AG, Basel

\section{Introduction}

We feel this case is important because it catalogs discovery of a very rare pathology. In addition, we present an instance of radiographic findings demonstrating consistent and progressive leptomeningeal enhancement, thus adding further weight to the idea that imaging alone should be enough for diagnosis. Lumbar punctures (LP) have poor sensitivity when compared to imaging and a leptomeningeal biopsy is invasive; plus it carries the risk of malignant seeding. Time is precious with this diagnosis because the treatment options are limited, invasive, and prognosis is already grim. The most common locations of metastasis from the prostate are bone, lung, and liver [1]. Leptomeningeal spread occurs more with hematologic tumors, such as leukemia, at an incidence of $10-15 \%$, and much less frequently with solid tumors at an incidence of 1-5\%, the most common being breast, lung, melanoma, gastrointestinal, and primary central nervous system tumors [2, 3]. This difference is also relevant in nomenclature, as hematologic malignancies with metastasis to the meninges are referred to as lymphomatous meningitis while solid tumors are referred to as leptomeningeal carcinomatosis (LC) [4, 5]. An example of the prevalence of LC can be provided by a 33-year retrospective review published by the University of Texas MD Anderson Cancer Center. The study aimed to detect all patients with LC originating from primary GU cancer. Of the 93,960 GU cancer patients, a mere 31 cases of LC were identified; approximately $0.03 \%$. Of those that were identified, only 7 were prostate in origin [6]. The prognosis was such that most died with bone metastasis, nodal disease, or less commonly, visceral metastases. This case adds to the growing body of evidence that imaging can be diagnostic for LC.

\section{Case Presentation}

We present a 65-year-old male with a past medical history of hypothyroidism, restless leg syndrome, and established stage 4 prostate cancer with metastasis to the liver and bone who completed radiation and chemotherapy. He presented with gradually worsening, generalized weakness for 2 weeks, associated with multiple episodes of non-bilious, non-bloody vomiting for 1 week before admission. He had no sick contacts, fever, cough, abdominal pain, dysuria, or recent travel history. The remaining review of systems was unremarkable. The patient's prostate cancer diagnosis was confirmed by biopsy in June 2017 (Gleason score of 9), and he started his medical management with leuprolide. Over the next 2 years after diagnosis, he was effectively treated with the following medications: abiraterone, cabazitaxel, radium-223, and enzalutamide.

Vital signs were unremarkable during admission and throughout his hospitalization. Physical examination, including a complete central nervous system exam, was unremarkable, with intact cranial nerves and without neurologic deficits. He did not appear cachectic. Laboratory workup on admission showed: white blood cell count of 2.5, hemoglobin of 9.7, corrected calcium of 6.6 , potassium of 3.1, alkaline phosphatase of 221, prostate-specific antigen of 182, and ferritin level of 1,336.

\section{Karger'}




\section{Case Reports in Oncology}

\begin{tabular}{l|l}
\hline Case Rep Oncol 2021;14:753-760 \\
\hline DOI: 10.1159/000514755 & $\begin{array}{l}\text { @ 2021 The Author(s). Published by S. Karger AG, Basel } \\
\text { www.karger.com/cro }\end{array}$ \\
\hline
\end{tabular}

Stenberg et al.: Leptomeningeal Carcinomatosis of Metastatic Primary Prostate Cancer

Fig. 1. Transverse view: Superior slice showing enhancement in the middle cranial fossa of the anterior right temporal lobe.

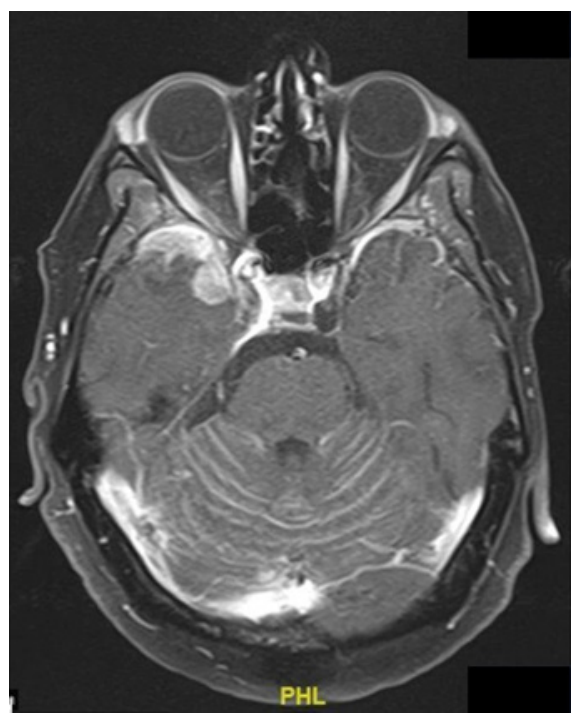

Fig. 2. Transverse view: Inferior slice showing enhancement in the middle cranial fossa of the anterior right temporal lobe.

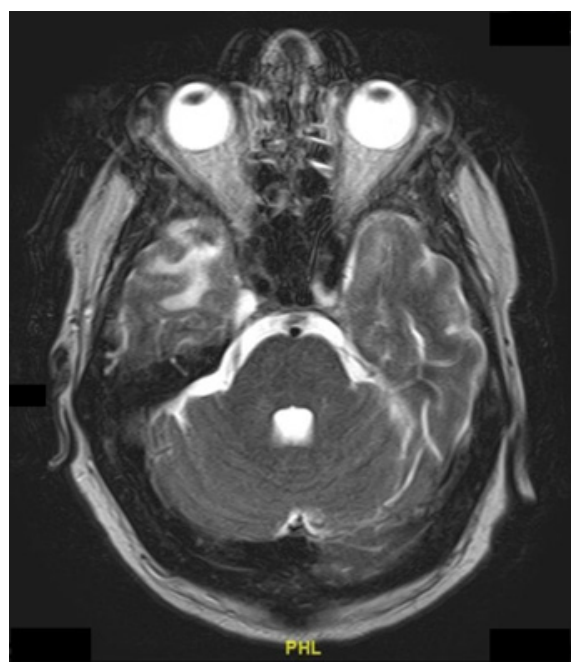

Fig. 3. Coronal view: enhancement seen in the middle cranial fossa of the anterior right temporal lobe.

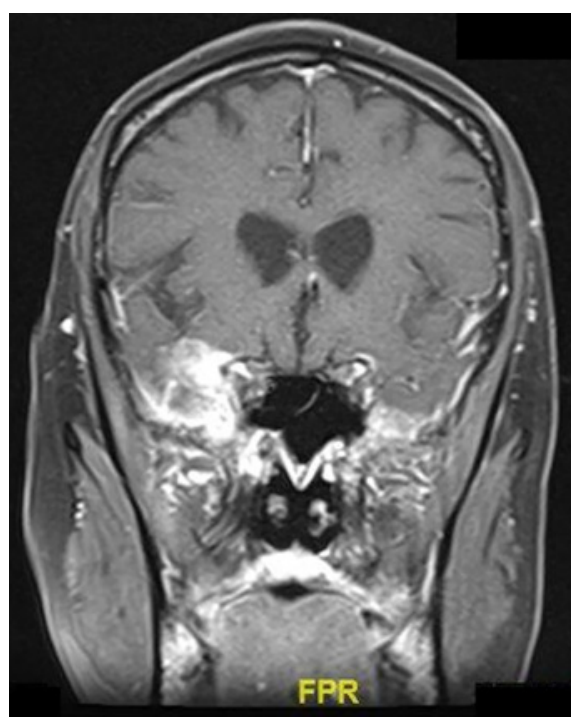


MRI of the brain with contrast showed bilateral temporal lobe edema with middle cranial fossa dural thickening/enhancement. An abnormal enhancement extended to the margins of both orbital apices and right cavernous sinus. Leptomeningeal enhancement in the posterior fossa was also noted (Fig. 1-3). CT head with contrast showed irregular, nodular dural thickening and enhancement related to the right temporal region with adjacent temporal and frontal lobe edema. CT of the chest/abdomen/pelvis showed multiple sclerotic lesions throughout the pelvis, lumbar spine, thoracic spine, and ribs consistent with metastatic disease. Multiple 1-2 cm masses in the liver were noted. Diagnostic LP was performed to evaluate for leptomeningeal spread of prostate cancer. Cerebrospinal fluid (CSF) analysis showed normal RBC, WBC, and glucose; however, elevated total protein level and atypical cells consistent with adenocarcinoma were seen. Upon discharge, the patient was seen as an outpatient and started on olaparib. He continued olaparib, but did not remain in the area and hence was not offered intrathecal chemotherapy (ITC).

\section{Discussion}

\section{Differential Diagnoses}

Our patient had limited differentials to begin with, as he had known metastatic disease. It is important to differentiate LC from parenchymal disease as $30-40 \%$ of those with LC have brain metastasis, too. Sarcoid, spinal metastasis, and various types of meningitis are also potential differentials. The differential is critical as the median overall survival for intradural extramedullary spinal metastasis is significantly higher, 732 days, as compared to patients with LC, 35 days, according to Knafo et al. [7].

\section{Pathology}

Leptomeninges is the name given to the pia and arachnoid mater, two of the layers surrounding the brain and spinal cord. The subarachnoid space contains CSF in addition to many arteries that penetrate into the parenchyma of the brain. This unique anatomical arrangement is how tumor cells invade the leptomeninges. The two most common routes of tumor extension into the CSF are either hematogenous spread via arachnoid vessels or direct extension from the brain parenchyma.

The pathology of LC is a manifestation of increased ICP either from mass effect due to bulky tumors or impedance of CSF. The presentation of clinical signs can be due to cranial nerve and spinal root dysfunction, direct invasion of the brain parenchyma by tumor cells resulting in competition for essential metabolites, or disruption of the blood-brain barrier by cancer cells. This disruption occurs via upregulation of complement component 3 leading to penetration of plasma growth factors into the CSF [2]. The resulting symptoms of LC occasionally manifest insidiously but it is often the complication of more advanced cancers. Typical presenting symptoms include altered mental status, headache, nausea, vomiting, and cranial nerve palsies. Our patient presented with lower extremity weakness, spasms, nausea, and vomiting [4, 8]. Common symptoms seen in patients are sequelae of increased ICP, such as headache, nausea, and vomiting, and gait disturbances, as demonstrated in our patient who presented with several days of nausea, vomiting, generalized weakness, and diarrhea [6]. Our patient was also known to have diffuse spinal involvement and had been experiencing worsening lower extremity weakness and restless leg syndrome, likely as a result of spinal nerve root involvement. In one series of 150 patients with solid tumor metastases, of which only one was prostate, and with an average Karnofsky score of 70, the most common presenting signs were headache at 39\%, nausea and vomiting at $25 \%$, leg weakness at $21 \%$, cerebellar dysfunction at $17 \%$, altered mental status at $16 \%$, diplopia at $14 \%$, and facial weakness at $13 \%$ [9].

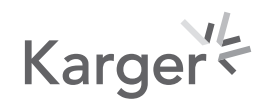




\section{Diagnosis}

Diagnosis is challenging given the poor sensitivity of diagnostic modalities. Following detailed physical examination, the patient should have a contrast MRI. However, the reported sensitivity is highly variable across the literature ranging from 77 to $100 \%$. As in our patient, enhancement of the leptomeninges may be found in addition to hydrocephalus and subependymal nodules, deposits, etc. Logically, the next step would be an LP. The most definitive tool in the diagnosis of LC is the LP, searching for cytologic identification of malignant cells in the CSF [5]. Our patient was not a candidate for an LP on a previous admission, approximately 1 month earlier, due to mild thrombocytopenia; however, his platelets had recovered enough for an LP on the present admission. Unfortunately, CSF cytology can have false-negative rates as high as $35 \%$. If clinical suspicion remains high following a negative LP, repeat testing is encouraged and approaches a sensitivity of $85-90 \%$ on the third attempt. CSF fibronectin and beta-glucuronidase levels are also other predictors of LC [10]. The first LP on our patient was positive for atypical cells imparting a signet ring morphology, consistent with adenocarcinoma. Furthermore, our patient had mild hypoglycorrhachia (usually less than $60 \mathrm{mg} / \mathrm{dL}$ ) at $50 \mathrm{~mL} / \mathrm{dL}$, and elevated protein (usually greater than $45 \mathrm{mg} / \mathrm{dL}$ ) at 104 . Both of these findings have been seen in cases of LC [2].

In patients without a known cancer diagnosis, capturing a new diagnosis of LC can be challenging and in addition to LPs, the patient may need an open biopsy of the leptomeninges. Capturing an accurate diagnosis is very important, as there are many differentials in LC such as neoplasms, meningitis, Lyme, or West Nile virus. Artifacts seen on imaging can skew diagnoses as seen in post-radiotherapy nerve root thickening, post-LP changes, or enhancing meningeal vasculature.

\section{Treatment}

Strategies in fighting LC are dependent on the patient's Karnofsky Performance Score (KPS), a frequently utilized scoring tool with high validity. Our patient scored a KPS of approximately 60 with mixed features both above and below 60 . This is significant because the determination of "good risk" versus "poor risk" occurs at 60. A patient who is deemed good risk is a candidate for treatment of the entire neuraxis because limited therapy may result in extension of cancer to untreated sites. Our patient was a candidate for an aggressive approach to treatment. His MRI was without evidence of bulky disease but rather linear enhancement and only focal neurologic symptoms. Grade 2C evidence recommendations are ITC rather than radiotherapy. The preferred agent is intrathecal methotrexate for individuals with solid tumor metastasis as in our patient, with grade $2 \mathrm{~B}$ evidence. The starting regimen is $10-12 \mathrm{mg}$ bi-weekly for 4 weeks with a taper to weekly and then every other week in those showing a response. To prevent myelosuppression and systemic toxicity, there is grade $2 \mathrm{C}$ evidence for oral leucovorin $10 \mathrm{mg}$ orally twice a day for 3 days. Clinical signs, symptoms, and frequent measurements of tumor markers are used to assess for responsiveness to treatment. Failure to clear malignant cells from CSF within 1-2 months would necessitate seeking alternating therapies and or re-evaluation of treatment goals.

\section{Conclusion}

LC remains difficult to diagnose given insensitivity of CSF studies. Enhancements in MRI have tremendously increased the accuracy of the diagnostic process but controversy remains over initiating treatment, specifically ITC, without direct biopsy. CSF studies are commonly performed, though noted to have poor sensitivity. Often, repeat CSF studies are performed when clinical suspicion remains high. Our patient had initially presented approximately 1

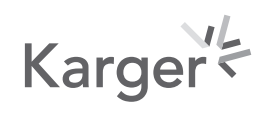


month prior with similar symptoms and imaging; however, on initial encounter, he was thrombocytopenic and could not consent to an LP. On his subsequent admission and after resolution of thrombocytopenia, an LP was performed, and he was noted to have positive cytology for adenocarcinoma. Despite such a high false negative percent, 35\%, his first LP happened to yield a positive result, albeit greater than a month after initial presentation. The patient received MRIs on both admissions, which demonstrated consistent findings showing progression on the latter study, raising the question of reliability of an LP versus brain MRI.

Evidence points to MRI imaging in multiple studies, having a sensitivity of $87 \%$. It is noninvasive, safer, and more readily available. By contrast, the sensitivity of cytology is approximately $54-85 \%$ and can have repeated false negatives, as much as $34 \%$, in patients with clinically or radiographically unequivocal disease. This is thought to be due to the propensity of solid tumor metastasis to adhere to neural structures and form detectable nodules rather than exist, free-floating, in the cerebrospinal fluid. The sensitivity may approach $90 \%$, but on the third attempt, thus subjecting the patient to pain and other associated risks, multiple times. On the other hand, cell cytology, when positive, has a very high specificity, as a false positive is very rare in the hands of an experienced cytopathologist [11].

Conflicting evidence exists for pursuit of dual diagnostic methods as well as initiating treatment following one diagnostic method. The rate of diagnoses of LC utilizing neuroimaging alone was approximately $45 \%$ in Clarke et al.[9], demonstrating the effectiveness of neuroimaging when compared with cell cytology. While both imaging and LP are known to be diagnostic and will increase diagnostic accuracy, an LP is not always necessary when there are definitive findings on imaging to establish diagnosis [12].

We find it difficult to support dual pursuit of diagnoses via imaging and cytology in all cases given the precarious nature of results in cytology and the relative precision with neuroimaging. Upon review of the available literature, we feel that for our patient, a risk benefit analysis may have been in favor of ITC based on MRI findings and without a cytology diagnosis. If ITC had been initiated on his previous visit, a month prior, given positive history, symptoms, and imaging finding, his life expectancy and quality may have been positively impacted. Various treatment modalities, including ITC would have been offered. Given the median survival length from diagnosis, the interval of 30 days likely represented a quarter to a third of his remaining life expectancy. After reviewing available evidence, we support a case-by-case application of diagnostic modalities, especially in patients similar to ours. Given our patient's known history of metastatic prostate cancer, and consistent, progressive neuroimaging findings, imaging alone should have been viewed as a gold-standard-yielding mode of diagnosis.

Our patient had previously been engaged in therapy; however, his prognosis continued to worsen with continuing metastasis. He was visiting the area during the time of this hospitalization and subsequently returned home. His records were made available to his oncologist and he was likely counseled on ITC. The prognosis of LC is poor, as mentioned previously, due to the rapid decline. There is a paucity of randomized controlled trials and so treatment is derived from expert opinions and lower evidence studies. As is common, radiation followed by ITC is helpful for large tumor burden. If present and symptomatic, CSF flow obstruction is relieved via the appropriate intervention, bearing in mind that this can convey risk of seeding malignant cells in new locations. An often underlooked part of care at this stage is palliative and supportive treatment and psychostimulants, modalities that should invariably be considered [2].

The approach to treating LC is done best by an interdisciplinary team, and quality of life should be a focal point without exception. Until not too long ago, patients were treated with androgen deprivation, radiotherapy, and surgery for locally advanced tumors; the results were poor and varied in nature. Newer biologic agents have entered the conversation; however, they have only been shown to have a mild effect on prolongation of life for patients with LC, and they often burden the family with the immeasurable cost of the medications.

\section{Karger' ${ }^{\prime \prime}$}




\section{Statement of Ethics}

Written informed consent for publication was obtained from the patient's next of kin, as the patient was deceased. She consented to the publication of this case report and any accompanying images. The consent form will be made available to the editor if requested.

\section{Conflict of Interest Statement}

The authors declare that there is no conflict of interest regarding the publication of this paper.

\section{Funding Sources}

This case report did not receive grants from any funding agency in the public, commercial, or not-for-profit sectors.

\section{Author Contributions}

Dr Stenberg wrote significant parts of the manuscript, reviewed the data provided, and participated in patient care. He drafted the work for important intellectual content and provided the extra-final approval of the revision to be published. He agreed to be accountable for all aspects of the work in ensuring that questions related to the accuracy or integrity of any part of the work are appropriately investigated and resolved. Dr Shalavadi wrote and reviewed significant parts of the manuscript, provided substantial contribution to the conception or design of the work, revised the manuscript critically for important intellectual content, and gave the final approval of the final version to be published. She agreed to be accountable for all aspects of the work in ensuring that questions related to the accuracy or integrity of any part of the work are appropriately investigated and resolved. Dr Syeda wrote and reviewed some parts of the manuscript, provided contribution to the conception or design of the work, revised the manuscript critically for important intellectual content, and gave the final approval of the final version to be published. She agreed to be accountable for all aspects of the work in ensuring that questions related to the accuracy or integrity of any part of the work are appropriately investigated and resolved. Dr Reddy wrote and reviewed some parts of the manuscript, provided contribution to the conception or design of the work, revised the manuscript critically for important intellectual content, and gave the final approval of the final version to be published. He agreed to be accountable for all aspects of the work in ensuring that questions related to the accuracy or integrity of any part of the work are appropriately investigated and resolved. Dr Saunders reviewed parts of the manuscript, provided substantial contribution to the conception or design of the work, revised the manuscript critically for important intellectual content, partook in direct patient care, and gave the final approval of the final version to be published. As the attending physician, he agreed to be accountable for all aspects of the work in ensuring that questions related to the accuracy or integrity of any part of the work are appropriately investigated and resolved.

\section{Karger' ${ }^{\prime \prime}$}




\section{References}

1 Cone LA, Koochek K, Henager HA, Fausel R, Gade-Andavolu R, Potts BE, et al. Leptomeningeal carcinomatosis in a patient with metastatic prostate cancer: case report and literature review. Surg Neurol. 2006;65(4): 372-6.

2 Batool A, Kasi A. Leptomeningeal Carcinomatosis. [Updated 2020 Apr 14]. In: StatPearls [Internet]. Treasure Island (FL): StatPearls Publishing; 2020 Jan-. Available from: https://www.ncbi.nlm.nih.gov/books/ NBK499862/

3 Carroll RD, Leigh EC, Curtis Z, Thorpe A, Ballengee J, Pacioles T. A Case of Leptomeningeal Carcinomatosis from Aggressive Metastatic Prostate Cancer. Case Rep Oncol. 2019 Jan-Apr;12(1):311-6.

4 Wang N, Bertalan MS, Brastianos PK. Leptomeningeal metastasis from systemic cancer: review and update on management. Cancer. 2018 Jan;124(1):21-35.

5 Grossman SA, Krabak MJ. Leptomeningeal carcinomatosis. Cancer Treat Rev. 1999 Apr;25(2):103-19.

6 Yust-Katz S, Mathis S, Groves MD. Leptomeningeal metastases from genitourinary cancer: the University of Texas MD Anderson Cancer Center experience. Med Oncol. 2013;30(1):429.

7 Knafo S, Pallud J, Le Rhun E, Parker F, Iakovlev G, Roux FX, et al. Intradural extramedullary spinal metastases of non-neurogenic origin: a distinct clinical entity or a subtype of leptomeningeal metastasis? A case-control study. Neurosurgery. 2013 Dec;73(6):923-32; discussion 932.

8 Bernstein WB, Kemp JD, Kim GS, Johnson VV. Diagnosing Leptomeningeal Carcinomatosis With Negative CSF Cytology in Advanced Prostate Cancer. J Clin Oncol. 2008;26(19):3281-4.

9 Clarke JL, Perez HR, Jacks LM, Panageas KS, Deangelis LM. Leptomeningeal metastases in the MRI era. Neurology. 2010;74(18):1449-54.

10 Schneck MJ. Leptomeningeal Carcinomatosis Differential Diagnoses. Medscape.com. https://emedicine. medscape.com/article/1156338-differential. Published August 6, 2020. Accessed September 12, 2020.

11 Glantz MJ, Cole BF, Glantz LK, Cobb J, Mills P, Lekos A, et al. Cerebrospinal fluid cytology in patients with cancer: minimizing false-negative results. Cancer. 1998 Feb 15;82(4):733-9.

12 Straathof CS, de Bruin HG, Dippel DW, Vecht CJ. The diagnostic accuracy of magnetic resonance imaging and cerebrospinal fluid cytology in leptomeningeal metastasis. J Neurol. 1999;246(9):810. 